\title{
A Novel Emergency Message Broadcast Game based on Link Quality in Vehicular ad hoc networks
}

\author{
Xi Hu, a , XingGuo Cheng ${ }^{2, b}$ \\ ${ }^{1}$ Computational center of Northeastern University at Qinhuangdao, Northeastern \\ University at Qinhuangdao, Qinhuangdao, 066004, China \\ ${ }^{2}$ Information Institute of Northeastern University, Northeastern University, \\ Shenyang, 110819, China \\ aemail: neuqhx @126.com, bemail:xiaoxing610@139.com
}

Keywords: VANETs; Broadcast Scheme; Game Theory; Nakagami RF model

\begin{abstract}
In vehicular ad hoc networks, the typical problem broadcast storm always happen when vehicles are usually located quite densely within the range of radio transmission. In the paper, a novel emergency message broadcast game is proposed and the game takes nodes' density and link quality into account, each in-between neighbor node can calculate the value of link quality using node's receiving power and by Nash equilibrium determine its probability for rebroadcasting the received emergency message independently. NS-2 simulator is used to evaluate the performance of the proposed scheme, the simulation results show that this proposed broadcast scheme is effective , which can reduce broadcast overhead and decrease transmission delay obviously.
\end{abstract}

\section{Introduction}

Vehicular Ad hoc Networks (VANETs) have been rapidly attracting interest from both academic and industrial communities for the sake of their importance in Intelligent Transportation System $(\mathrm{ITS})^{[1]}$. VANETs is a different form of Mobile Ad hoc Networks (MANETs) ${ }^{[2]}$ providing communication between vehicles in single hop or multi hops, and between vehicles and nearby fixed roadside equipment.

VANETs are expected to be able to significantly reduce the road accidents and improve traffic efficiency. However in VANETs, the vehicles' speed are fast, so the entire network topology changes are particularly fast and the network state will fluctuate dramatically. And the correct reception of the messages is not easy in the presence of obstacles and fading in the wireless channel. Thus it's a big challenge to completely design a suitable protocols for VANETs.

Due to above factors, a simple broadcast scheme can not work well. The simplest way to broadcast emergency messages is blind flooding $(\mathrm{BF})^{[3]}$ in which vehicle nodes always rebroadcast the messages vehicles received for the first or more times. However obviously, in a high-density network, each node rebroadcast the same message, it will cause a large amount of redundant information in the network, which leads to the collision and congestion and degrades the network's performance to achieve a low dissemination rate.

Emergency message warning for public safety is one of many applications that is highly time-critical and requires a more intelligent broadcast mechanism than just blind flooding. To mitigate the impact of the broadcast storm problem, several broadcast schemes have been proposed. In [4], N.Wisitpongphan and O.K.Tonguz have proposed three broadcast schemes: weighted p-persis- tence, slotted 1-persistence, and slotted p-persistence schemes. In these protocols, when a node receive a message, calculating a rebroadcast probability according to the distance from the sender node. Generally, a node with a larger distance from the sender node obtains a higher rebroadcast probability. Ozen K. Tonguz et.al. ${ }^{[5]}$ proposed a detailed implementation of the Distributed Vehicular Broadcast protocol. Their work focuses on highway scenarios and present the design and implementation of a new distributed vehicular multi-hop broadcast protocol, which can operate in all traffic regimes, including extreme scenarios.

Paper [6-8] have well studied broadcasting schemes based on position and distance in their 
works. PBAB, a Position-based Adaptive Broadcast, which is an event-driven protocol and suitable for cooperative collision avoidance in two-way multi-lane highway. The decisions that whether to relay messages are made for nodes' position, direction, and velocity ${ }^{[6]}$. In [7], a new broadcast method is proposed, which assures broadcasting of message to back and forward directions and selects furthest vehicles to rebroadcast message waiting a waiting time determined based on the distance, such that the waiting time is shorter for more distant nodes. TRAB for VANET called Transmission Range Adaptive Broadcast is proposed in [8]. Which considers the transmission ranges of vehicles changed adaptively and calculates different waiting time to select the relay nodes to ensure that less relay nodes will be chose to forward the emergency messages. Above approaches try to broadcast messages with low overhead, but as we will describe, these methods suffer from insufficiency that just take single factor into consideration to choose relay node to rebroadcast messages, especially distance or position.

Actually in VANETs, there are many parameters can be used to model the node and analyze network performance, however, nodes' inter distance、 the node's position and the relative velocity between nodes, directly or indirectly influences node's received power. The node's receiving power is larger that means link quality is better. Conversely, it means poorer link quality. So it's an optimized way to analyze node's receiving power compared to other parameters, because this is the most direct result to the communication link quality of the networks.

In this paper, we propose a novel message broadcast game (MBG). In this scheme, the rebroadcast of messages is modeled with a game in which nodes' density and link quality are taken into account, each in-between neighbor node can calculate the value of link quality using node's receiving power and determine its probability for rebroadcasting the received emergency message independently.

\section{Design of the Message Broadcast Game}

In this part, we introduce our propose message broadcast game (MBG). Section $A$ presents the mix strategy game used in the paper and use it to model node's actions. Section $B$ describes about the channel model and node's power model. Section $C$ presents the broadcast scheme about how to combine game theory and node's receiving power. This game takes nodes' density and node's receiving power into account, each node calculate the value of link quality using node's receiving power and determine its probability for rebroadcasting the received emergency message.

\section{A. Game Theory}

In [9] [10], a mix strategy game named "reporting a crime" is introduced. In this game, a crime is observed by a group of $n$ people. Each person would like the police to be informed, but prefers that someone else make the phone call. Specifically, suppose that each person attaches the value $R$ to the police being informed and bears the cost $C$ if he/she makes the phone call, where $R>C>0$. The situation is modeled by the strategic game with vNM preference. Then we can model our broadcast scheme using above strategic game, as following:

- Players: The $n$ nodes including node $S$ and its $n-1$ neighbor nodes

- Actions: Each node's set of action is \{Rebroadcast the message, Don't Rebroadcast the message $\}$.

- Preferences: Each node’s preferences are represented by the expected value of a payoff function that assigns 0 to the profile in which no node rebroadcasts the message, $R^{-} C$ to any profile in which only node $S$ rebroadcasts the message, and $R$ to any profile in which at least one node rebroadcasts the message, but node $S$ does not.

So we set up a theoretical model of a process for nodes whether to rebroadcast the message. Analyzing the model, it has a symmetric mixed strategy equilibrium in which each node rebroadcast the message with the positive probability $p$ less than 1 . In any such equilibrium, each node's expected payoff to rebroadcast is equal to its expected payoff to not rebroadcast. Each node's payoff to rebroadcast is $R^{-} C$, and its payoff to not rebroadcast is 0 if no one else broadcasts and $R$ if at 
least one other neighbor node rebroadcasts, so the equilibrium condition is:

$$
R-C=0 * \operatorname{Pr}\{\text { no node rebroadcast }\}+r * \operatorname{Pr}\{\text { at least one node rebroadcast }\}
$$

or

$C / R=\operatorname{Pr}\{$ no one else rebroadcast the message $\}$

Denote by $p$ the probability with which each node rebroadcast. The probability that no one else rebroadcast is the probability that every node of the other $n^{-1}$ nodes do not rebroadcast, as $(1-p)^{n-1}$. Thus, the equilibrium condition is as following:

\section{B. Power Model}

$$
p=1-\left(\frac{C}{R}\right)^{1 / n-1} \text {. }
$$

As an addition to the existing radio propagation models, Nakagami RF model is developed and suitable for VANETs channel. Nakagami is a mathematical general modeling of a radio channel with fading. Compared to the existing models (two-ray ground and shadowing), Nakagami RF model has more configurable parameters to allow a closer representation of the wireless communication channel. It is able to model from a perfect free space channel, to a moderate fading channel on highway, even to a dramatically fading channel in urban communities.

Nakagami distribution is defined by the following probability density function:

$$
f(x)=\frac{2 m^{m} x^{2 m-1}}{\Gamma(m) \Omega^{m}} \exp \left[-\frac{m x^{2}}{\Omega}\right], x \geq 0, \Omega>0, m \geq \frac{1}{2},
$$

Parameter $x$ is the distance far from the source node, parameter $\Omega$ is the expected value of the distribution and can be interpreted as the average received power. $\Gamma(m)$ is gamma distribution about parameter $m$ and $m$ is the so called shape or fading parameter.

The corresponding pdf of power (square of the signal amplitude) at the given distance can be obtained by a change of variables and is given by a gamma distribution of the following form:

$$
p_{r}(x)=\left(\frac{m}{\Omega}\right)^{m} \frac{x^{m-1}}{\Gamma(m)} \exp \left[-\frac{m x}{\Omega}\right], x \geq 0 .
$$

$p_{r}(x)$ is node's receiving power with parameter $x$.

\section{Details of Designed Broadcast Scheme}

Here, we combine above game theory and power model, and proposed a variable LNQ ( Link Quality )representing the communication link quality between nodes in this paper as following:

$$
L N Q=\sqrt{\frac{p_{t}}{p_{r}^{*} d}},
$$

Especially, Pt $、 P r$ and $d$ represent the power node transmitted the power node received and the average distance between node and other neighbor nodes respectively. The transmission power of all the vehicles are same as default in the simulation. The above three factors are associated mutually, so we can analyze and know that $L N Q$ is monotonically increasing with the increase $d$.

Thus, we define two functions $C$ and $R$, which both are the function about $L N Q, C$ is a cost function which denotes the cost paid by node when it rebroadcast the message; $R$ is a reward function which denotes the reward obtained by node when any other nodes rebroadcast the message.

Furthermore, $C$ is a monotonic decreasing function about $L N Q$ which means that the more the communication quality is, the less costs paid, and $R$ is a monotonic increasing function about $L N Q$ which means that the more the communication quality is, the more rewards obtained. So $C$ / $R$ is monotonic decreasing with the increasing $L N Q$. Thus we define $p$ as following:

$$
p(n, L N Q)=1-\left(\frac{1}{1+L N Q}\right)^{a / n-1} \text {. }
$$


In the model, the probability for nodes to rebroadcast the message increases with the increasing of the $L N Q$, and decreases with the increasing of the number of neighbor nodes. Parameter $a$ is the adjustment factor and certainly obtain appropriate value for simulations. The details of MBG scheme are shown in Fig. 1.

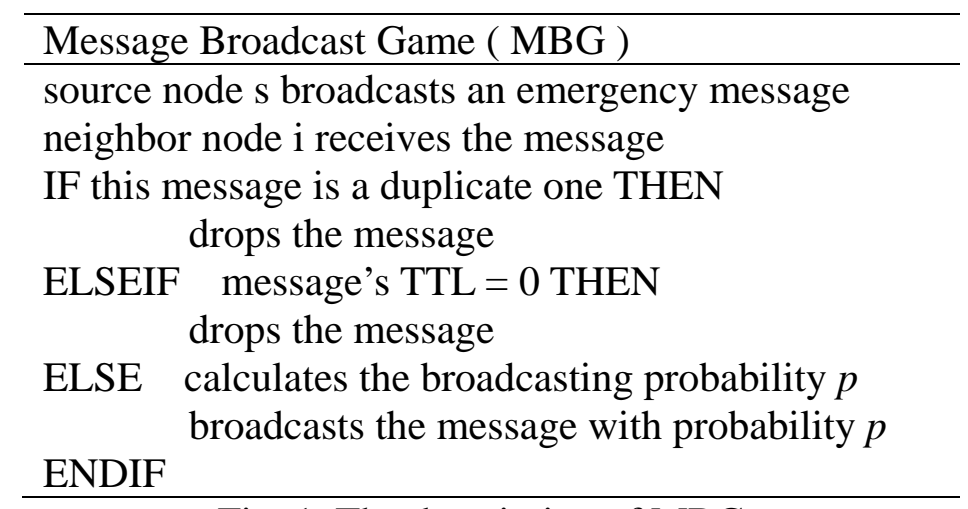

Fig. 1. The description of MBG

\section{Test results}

We put the networks simulation into NS2.35, and the main work is to evaluate the performance of BF algorithm and MBG algorithm based on simulation. In our simulations, basic simulation parameter settings as following as Table 1 . Node neighbor numbers v node transmit power and node received power which are used to evaluate the quality of link and communication. The evaluation metrics are shown as following.

(1)Application request: The total number of application agent request for data packets delivered to the nodes in the VANETs.

(2) Broadcast overhead: The ratio of the number of all drop packets in the total number of data packets.

(3) Average delay: The expected value of the delay incurred by all data packets that originate at the source and are delivered to the destination.

Table 1. Parameter settings

\begin{tabular}{ll}
\hline Parameter & Value \\
\hline Number of vehicles & $10,30,50,70,100$ \\
Velocity & Min: $5 \mathrm{~m} / \mathrm{s}$, Max:34 m/s \\
Scene & $2000 \mathrm{~m} \times 2000 \mathrm{~m}$ \\
Radio propagation model & Nakagami RF model \\
Packet Delivery Rate & 1 packet/sec \\
Transmission Range & $250 \mathrm{~m}$ \\
Simulation Time & $400 \mathrm{~s}$ \\
Parameter $a$ & 10 \\
\hline
\end{tabular}

The results are shown in Figure.2. From Fig.2.a, The number of application requests of BF protocol is almost similar to MBG protocol, and both fluctuate in the 1300 times. As shown in Fig.2.b, the routing overhead of two broadcast algorithms all increase as the node's number increases. BF protocol has bigger routing overhead than MBG protocol, since the in-between nodes of BF need to forward identical packets more than once. However, because game is used in MBG, which can effectively limited the number of identical packet forwarding, the routing overhead of MBG is clearly less than BF, and the superiority increases as the node's number increases.

As shown in Fig.2.c, the average delay of two algorithms all increase as the node's number increases, and the rate of increasing is faster when the node's number increases from 10 to 50 than that when the node's number exceeds 50 . Moreover, it also shows that BF has the biggest delay than MBG. The reason is that more packet receives and rebroadcasts induce more waiting time and 
collisions at MAC layer. So it results to data packets queue in in-between nodes for longer time.

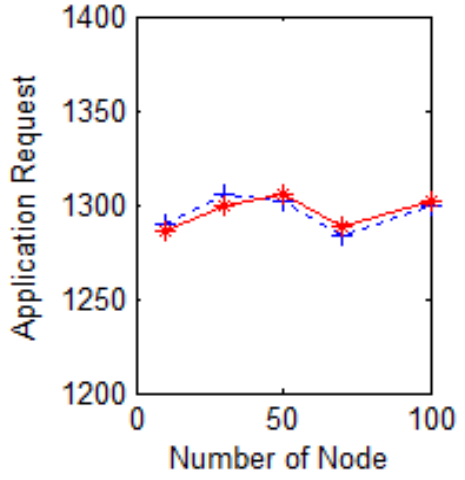

(a)

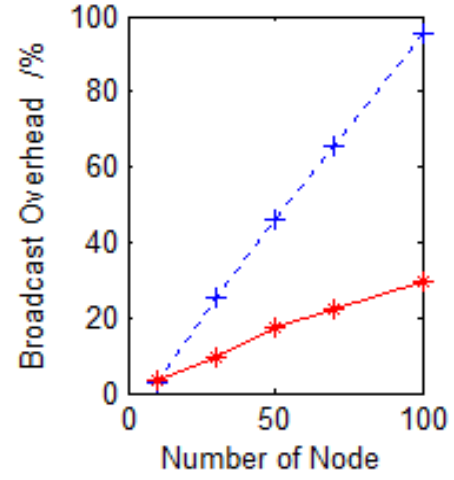

(b)

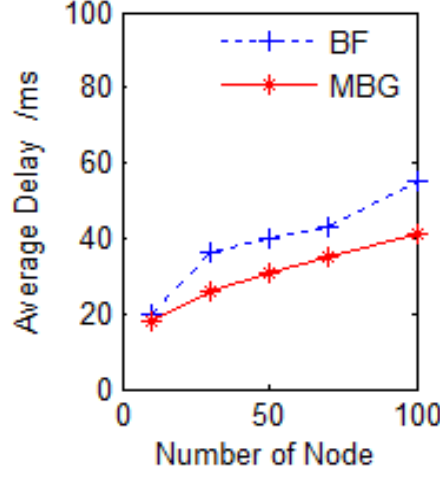

(c)

Fig.2. The metrics analyzed with different node's number

Although the MBG broadcast scheme through the Hello packet periodically to obtain the node's location and received power, there is a certain amount of routing overhead, but the Hello packet is smaller than other data packet. When the number of node increases, the routing overhead Hello packet induced compare with the data packet is still small.

\section{Conclusion}

In vehicular ad hoc networks, broadcast storm may happen if nodes rebroadcast the identical packet received more than once. Based on the analysis of the game theory, designed broadcast protocol can decrease the number of message forwarding and promote the performance of the networks. Experiments show that the new designed broadcast scheme MBG has less routing overhead and less average delay than BF, thus the designed broadcast scheme has higher efficiency and reliability.

As further research, we intend to theoretically analyze the value of parameter $a$ and the broadcast efficiency of the network. Enhancing the proposed broadcast scheme to adapt to a particular scene, as highway or urban scenarios.

\section{Acknowledgement}

In this paper, the research was sponsored by the National Natural Science Foundation of China (Grant No. 61501102) and Natural Science Foundation of Hebei Province, China (Grant No. F2014501082).

\section{References}

[1] Wu, Celimuge, S. Ohzahata, and T. Kato. Fuzzy Logic Based Multi-Hop Broadcast For HighDensity Vehicular Ad Hoc Networks. Vehicular Networking Conference (VNC), 2010 IEEE 2010:17 - 24.

[2] Gandhi, M., \& Khan, M.A. Performance analysis of metrics of broadcasting protocols in VANET. Computational Intelligence on Power, Energy and Controls with their impact on Humanity (CIPECH). 2014. Innovative Applications of IEEE. pp.315-321.

[3] Aravindhan, K., Kavitha, G., \& Dhas, C.S.G. Plummeting data loss for multihop wireless broadcast using position based routing in VANET. Science Engineering and Management Research (ICSEMR), 2014 International Conference on IEEE.

[4] Wisitpongphan, N., et al. Broadcast storm mitigation techniques in vehicular ad hoc networks. Wireless Communications IEEE 14.6, 2008:84-94.

[5] Tonguz, O. K., Wisitpongphan, N., \& Fan, B. Dv-cast: A distributed vehicular broadcast 
protocol for vehicular ad hoc networks. IEEE Wireless Communications, 2010: 17(2), 47-57.

[6] Yang, Y. T., \& Chou, L. D. Position-Based Adaptive Broadcast for Inter-Vehicle Communications. Communications Workshops, 2008. ICC Workshops '08. IEEE International Conference on IEEE. pp.410-414.

[7] Khakbaz, M Fathy. Adding Reliability of Broadcast Methods in Vehicular Ad hoc Networks. The Second International Conference on Next Generation Mobile Applications, Services, and Technologies, 2008.

[8] Wu, X. W., Yan, W., Song, S. M., \& Wang, H. B. A Transmission Range Adaptive Broadcast Algorithm for Vehicular Ad Hoc Networks. Networks Security Wireless Communications and Trusted Computing (NSWCTC), 2010 Second International Conference on IEEE. Vol.1,pp.28-32.

[9] Martin J. Osborne, An introduction to game theory, 2004, Oxford University Press. Inc., pp: 131-132.

[10] Hu, X., Wang, C., Song, X., \& Wang,. Stability-based rreq forwarding game for stabilityoriented route discovery in manets [J]. Wireless Personal Communications, 2012.68(4), 1689-1705. 